\title{
UNSOLVABLE DIOPHANTINE PROBLEMS
}

\author{
JULIA ROBINSON
}

We shall show that there is no general method of telling whether an arbitrary polynomial $P\left(x_{1}, \cdots, x_{k}\right)$ with integer coefficients is ever a power of 2 for $x_{1}, \cdots, x_{k}$ natural numbers. At present there is no general method known even in the special case with $k=1$.

Actually we shall show directly that the relation given by $r=2^{t}$ is diophantine in the set $\mathfrak{J}$ of powers of 2 . Hence every recursively enumerable set is diophantine in $J$ by Corollary 5 of Davis, Putnam, and Robinson [4].

We call a relation $\rho\left(x_{1}, \ldots, x_{n}\right)$ among natural numbers diophantine if there is a polynomial $P$ with integer coefficients such that $\rho\left(x_{1}, \cdots, x_{n}\right)$ if and only if there are natural numbers $y_{1}, \cdots, y_{k}$ with $P\left(x_{1}, \cdots, x_{n}, y_{1}, \cdots, y_{k}\right)=0$. A set $\delta$ (function $F$ ) is diophantine if $x \in \mathcal{S}$ (the graph of $F$ ) is diophantine. Also, $\rho$ is diophantine in $a$ set $S$ if there is a polynomial $P$ with integer coefficients such that $\rho\left(x_{1}, \cdots, x_{n}\right)$ if and only if there are natural numbers $y_{1}, \cdots, y_{k}$, $z_{1}, \cdots, z_{l}$ with both $P\left(x_{1}, \cdots, x_{n}, y_{1}, \cdots, y_{k}, z_{1}, \cdots, z_{l}\right)=0$ and $z_{1}, \cdots, z_{l} \in \mathcal{S}$. In [6], the term "existentially definable" was used instead of "diophantine". The definitions given there are easily seen to be equivalent to these. In both [1] and [4], relations over the positive integers were considered but the definitions and theorems hold for natural numbers with only the obvious modifications.

The logical symbols $\vee$ (there exists), $\wedge$ (and), $\leftrightarrow$ (if and only if) occur in this paper. The variables always range over the natural numbers.

Let $a>1$ and $a_{n}, a_{n}^{\prime}$ be defined by

$$
a_{n}+a_{n}^{\prime}\left(a^{2}-1\right)^{1 / 2}=\left(a+\left(a^{2}-1\right)^{1 / 2}\right)^{n} .
$$

Lemma 1. Natural numbers $x$ and $y$ satisfy the Pell equation $x^{2}-\left(a^{2}-1\right) y^{2}=1$ if and only if there is a natural number $n$ such that $x=a_{n}$ and $y=a_{n}^{\prime}$.

This is a standard result of elementary number theory.

Lemma 2. For $a>1$,

$$
a_{n}^{\prime} \equiv n \quad(\bmod a-1), \quad a_{n}-a_{n}^{\prime}(a-2) \equiv 2^{n} \quad(\bmod 4 a-5) .
$$

For proof, see Lemmas 5 and 7 of [6].

Presented to the Society, April 26, 1969; received by the editors September 18, 1968. 
LEMMA 3. There is a binary diophantine relation a such that $a(t, m)$ implies $m>2^{t}$ and if $m$ is sufficiently large with respect to $t$ then $a(t, m)$ holds.

Proof. Let $\psi$ be the relation of Lemma 8 of [6]. Then the binary relation $a$ defined by

$$
Q(t, m) \leftrightarrow(\bigvee u)(\psi(t+2, u) \wedge m>u)
$$

clearly satisfies the requirements.

Lemma 4. A natural number $n$ is the sum of two squares if and only if no number of the form $4 t+3$ divides $n$ to an odd power. Hence if $x$ and $y$ are relatively prime then $x y$ is the sum of two squares if and only if $x$ and $y$ are each the sum of two squares.

This is again a standard result of elementary number theory.

THEOREM 1. Let Th be an infinite set of natural numbers and $H$ be a diophantine function such that for all $m \in \mathfrak{T}$,

$$
(m, H(m))=1, \quad 2^{H(m)} \equiv 1 \quad(\bmod m) .
$$

Then $r=2^{t}$ is diophantine in $\mathfrak{T}$.

Before proving the theorem, we give two corollaries.

COROLLARY 1. Every recursively enumerable set is diophantine in any infinite set of numbers $m$ such that $2^{m-1} \equiv 1(\bmod m)$. In particular, $\mathfrak{T}$ can be any infinite set of primes.

Proof. From Theorem 1 with $H(m)=m-1$ and Corollary 5 of [4]. This result is also proved in [7].

COROLLARY 2. Every recursively enumerable set is diophantine in the set of numbers of the form $2^{2^{n}}$.

Proof. Let $\mathscr{T l}=\left\{2^{2^{n}}-1\right\}$ and $H(m)=m+1$. Now

$$
2^{2^{2^{n}}} \equiv 1 \quad\left(\bmod 2^{2^{n}}-1\right)
$$

since $2^{n}$ divides $2^{2^{n}}$. Hence for $m \in \mathscr{T}$,

$$
2^{m+1} \equiv 1 \quad(\bmod m)
$$

Hence the corollary follows from Theorem 1 as before.

REMARK. R. M. Robinson pointed out this application of the theorem to me.

Proof of Theorem 1 . We shall show that $r=2^{t}$ if and only if there are natural numbers $a, u, v$, and $m$ such that 
(1) $a>1, m \in \mathscr{N}, m|(4 a-5), H(m)|(a-1), m>1$,

(2) $a(t, m)$,

(3) $u^{2}-\left(a^{2}-1\right) v^{2}=1$,

(4) $\operatorname{Rem}(u-v(a-2), m)=r$,

(5) $\operatorname{Rem}(v, H(m))=t$.

Suppose that $a, u, v$, and $m$ satisfy (1)-(5). By Lemma 1 and (3), there is a natural number $n$ such that $u=a_{n}$ and $v=a_{n}^{\prime}$. Then by Lemma 2,

$$
\begin{array}{rlrl}
u-v(a-2) & \equiv 2^{n} & & (\bmod 4 a-5) \\
& \equiv 2^{n} \quad & (\bmod m)
\end{array}
$$

since $m \mid(4 a-5)$ by (1). Hence by (4),

(6) $r \equiv 2^{n}(\bmod m), r<m$.

Also by Lemma 2,

$$
\begin{aligned}
v & \equiv n & & (\bmod a-1) \\
& \equiv n & & (\bmod H(m))
\end{aligned}
$$

since $H(m) \mid(a-1)$ by (1). Hence by (5),

$$
t \equiv n \quad(\bmod H(m)), \quad t<H(m) .
$$

Thus, $n=t+q \cdot H(m)$ for some $q \geqq 0$. But by the hypothesis of the theorem $2^{B(m)} \equiv 1(\bmod m)$ and by $(2), A(t, m)$ holds so that

$$
2^{n} \equiv 2^{t} \quad(\bmod m), \quad 2^{t}<m .
$$

Hence by (6), $r=2^{t}$.

On the other hand, suppose $r=2^{t}$. We need to find $a, u, v$, and $m$ which satisfy (1)-(5). Choose $m$ in $\mathfrak{T}$ sufficiently large so that $a(t, m)$ holds. This is possible by Lemma 3 since $\mathbb{T}$ is infinite by hypothesis. Now $m$ is odd since $2^{H(m)} \equiv 1(\bmod m)$ and $H(m)>0$. Hence by the Chinese Remainder Theorem, we can choose $a>1$ so that

$$
4 a \equiv 5 \quad(\bmod m), \quad a \equiv 1 \quad(\bmod H(m))
$$

since $m$ and $H(m)$ are relatively prime. Hence (1) and (2) are satisfied. Now let $u=a_{t}$ and $v=a_{t}^{\prime}$ so that (3) holds. By Lemma 2, we have

$$
\begin{aligned}
u-v(a-2) & \equiv 2^{t} \quad(\bmod 4 a-5) \\
& \equiv 2^{t} \quad(\bmod m) .
\end{aligned}
$$

Also since $A(t, m)$ holds, $2^{t}<m$. Hence $\operatorname{Rem}(u-v(a-2), m)=2^{t}=r$ so that (4) holds. By Lemma 2 , 


$$
\begin{aligned}
v & \equiv t \quad(\bmod a-1) \\
& \equiv t \quad(\bmod H(m))
\end{aligned}
$$

since $H(m) \mid(a-1)$. Now $t<H(m)$ since $2^{t}<m$ and $2^{H(m)}>m$. Hence $\operatorname{Rem}(v, H(m))=t$ so (5) holds. Since all of the relations occurring in (1)-(5) except $m \in \mathscr{T}$ are diophantine, the theorem follows.

The following existential definition of the set of numbers of the form $2^{2^{n}}$ in terms of $J$, the set of powers of 2 , was suggested by the work of Davis [3].

TheOREM 2. $x=2^{2^{n}}$ for some $n>0$ if and only if $x$ is a power of 2 and there are natural numbers $u$ and $v$ such that $x=1+3\left(u^{2}+v^{2}\right)$.

Proof. Let $m$ be positive and $k$ be odd. Then

$$
2^{2^{m} \cdot k}-1=\left(2^{2^{m-1} \cdot k}+1\right)\left(2^{2^{m-2} \cdot k}+1\right) \cdots\left(2^{2 k}+1\right)\left(2^{2 k}-1\right) .
$$

Hence $\left(2^{2^{m} \cdot k}-1\right) / 3$ is the sum of two squares if and only if $\left(2^{k}-1\right)\left(2^{k}+1\right) / 3$ is the sum of two squares by Lemma 4 . (Note that $3 \mid\left(2^{k}+1\right)$ for $k$ odd.) Now for $k \geqq 3,2^{k}-1$ is of the form $4 t+3$ and is prime to $\left(2^{k}+1\right) / 3$ so the product is not the sum of two squares; while for $k=1$, it is. In the case $m=0$ and $k$ odd, $2^{k}-1$ is not divisible by 3 . Hence for odd $k, 2^{2^{m} \cdot k}=1+3\left(u^{2}+v^{2}\right)$ has a solution for $u$ and $v$ if and only if $m>0$ and $k=1$.

THEOREM 3. To every recursively enumerable set $\mathcal{S}$, there is a polynomial $P$ with integer coefficients such that

$$
\mathcal{S}=\left\{x: P\left(x, y_{1}, \cdots, y_{k}\right)=2^{t} \text { for some } y_{1}, \cdots, y_{k} \text {, and } t\right\} .
$$

Proof. Since $S$ is diophantine in $J$ by Corollary 2 and Theorem 2, there is a polynomial $F$ such that

$x \in \mathcal{S} \leftrightarrow$

$$
\left(\mathrm{V} u_{1}, \cdots, u_{m}, v_{1}, \cdots, v_{n}\right)\left(F\left(x, u_{1}, \cdots, u_{m}, 2^{v_{1}}, \cdots, 2^{v_{n}}\right)=0\right) .
$$

\section{Hence}

$$
\begin{aligned}
x \in \mathcal{S} \leftrightarrow & \left(\bigvee u_{1}, \cdots, u_{m}, w_{1}, \cdots, w_{n}, v\right) \\
& \left(F\left(x, u_{1}, \cdots, u_{m}, w_{1}, \cdots, w_{n}\right)=0 \wedge w_{1}\left|2^{v} \wedge \cdots \wedge w_{n}\right| 2^{v}\right) .
\end{aligned}
$$

We can now combine the conditions into a single polynomial in more variables in the usual way (see Davis $[1$, p. 104]). Thus,

$$
x \in S \leftrightarrow\left(\bigvee_{z_{1}}, \cdots, z_{r}, v\right)\left(G\left(x, z_{1}, \cdots, z_{r}, 2^{v}\right)=0\right)
$$

for a suitable choice of $G$. Finally, we see that 
$x \in S \leftrightarrow\left(\mathrm{V}_{z_{1}}, \cdots, z_{r}, w, v\right)\left(w\left(1+2 G\left(x, z_{1}, \cdots, z_{r}, w\right)^{2}\right)=2^{v}\right)$.

Hence we can take $w\left(1+2 G^{2}\right)$ for $P$.

REMARK. The arguments here are derived from those of Putnam [5] and Davis [2].

Since not every recursively enumerable set is recursive, Theorem 3 shows that there can be no general method of telling whether an arbitrary polynomial assumes a power of 2 as value.

Open Questions. Is every recursively enumerable set diophantine in the set of powers of 3 ? of $n$ ? Is there a general method of telling whether an arbitrary polynomial $P$ with positive integer coefficients assumes a power of 2 as value? (In this case, there is an obvious method of telling whether $P$ assumes a particular power of 2.)

\section{BIBLIOGRAPHY}

1. Martin Davis, Computability and unsolvability, McGraw-Hill, New York, 1958.

2. - Extensions and corollaries of recent work on Hilbert's tenth problem, I1linois J. Math. 7 (1963)', 246-250.

3. - One equation to rule them all, Memorandum RM-5494-PR, The RAND Corporation, Santa Monica, Calif., 1968.

4. Martin Davis, Hilary Putnam and Julia Robinson, The decision problem for exponential diophantine equations, Ann. of Math. 74 (1961), 425-436.

5. Hilary Putnam, An unsolvable problem in number theory, J. Symbolic Logic 25 (1960), 220-232.

6. Julia Robinson, Existential definability in arithmetic, Trans. Amer. Math. Soc. 72 (1952), 437-449.

7. - "Diophantine decision problems" in Studies in number theory, Studies in Math., vol. 6, Math. Assoc. of America; Prentice-Hall, Englewood Cliffs, N. J., 1969, pp. 76-116.

University of California, Berkeley 